\title{
Melting of Iron under Earth's Core Conditions from Diffusion Monte Carlo Free Energy Calculations
}

\author{
Ester Sola ${ }^{1}$ and Dario Alfè $\mathrm{e}^{1,2}$ \\ ${ }^{1}$ Thomas Young Centre@UCL, and Department of Earth Sciences, UCL, Gower Street, London WC1E 6BT, United Kingdom \\ ${ }^{2}$ London Centre for Nanotechnology, and Department of Physics and Astronomy, UCL, \\ Gower Street, London WC1E 6BT, United Kingdom \\ (Received 13 May 2009; published 14 August 2009)
}

\begin{abstract}
The temperature of Earth's core is a parameter of critical importance to model the thermal structure of Earth. Since the core is mainly made of iron, with a solid liquid boundary (the inner core boundary) at $1220 \mathrm{~km}$ from the center of the Earth, the melting temperature of iron at the pressure of the ICB provides constraints on the temperature of the core. These constraints are based either on extrapolations to ICB pressure of experimental measurements, or on theoretical calculations which employed various flavors of quantum mechanics, most notably density functional theory. Significant disagreement between estimates obtained with different methods calls for calculations based on more accurate techniques. Here we used quantum Monte Carlo techniques to compute the free energies of solid and liquid iron at ICB conditions. We obtained an iron melting temperature at $330 \mathrm{GPa}$ of $6900 \pm 400 \mathrm{~K}$.
\end{abstract}

PACS numbers: 91.35.- $\mathrm{x}, 02.70 . \mathrm{Ss}, 62.50 .-\mathrm{p}$

Knowing the thermal structure of Earth is of fundamental importance to our understanding of the dynamical mechanisms that govern the evolution of our planet, including the generation of the Earth's magnetic field in the Earth's core, and processes like plate tectonics and volcanism. Today we accurately know the density distribution inside the planet, and the pressure as function of depth. In particular, we know that the Earth's core is a solid ball of almost pure iron surrounded by a shell of liquid, also mainly formed by iron, extending up to $3480 \mathrm{~km}$ from the center [1]. We also know that a small fraction of light impurities like $\mathrm{O}, \mathrm{Si}$, and $\mathrm{S}$ must be present in the core. Very little is known about the temperature inside the Earth, as conventional probing techniques based on the analysis of seismic waves do not provide direct temperature measurements. The past two decades have witnessed strenuous efforts both on the experimental and the theoretical sides, towards what appear to be elusive attempts of measuring or computing the temperature of the Earth's core. These attempts exploit the presence of a boundary between the solid and the liquid core (the ICB), and therefore the fact that the core material must be at the melting point at that boundary. Since iron is the major component, experiments and theory have focused on the melting point of iron.

On the experimental side, the uncertainties are due to difficulties related to the extreme conditions, which require extrapolations from regions of much lower pressures and temperatures [2-9]. Theoretical calculations based on various approximations of quantum mechanics have been able to predict the whole melting curve of iron up to core pressures, but sometimes with conflicting results due to differences in the underlying techniques [10-13]. The most accurate calculations available to date are those based on density functional theory (DFT). In particular, in our earlier work, we used DFT to compute the free energies of solid and liquid iron in the whole range of core pressures, and obtained the melting curve by imposing equality of the Gibbs free energies of solid and liquid [10,11]. The melting temperature at $330 \mathrm{GPa}$ obtained with this method was $6350 \pm 600 \mathrm{~K}$ [11], where we estimated the first $\sim 300 \mathrm{~K}$ of the error being due to statistical sampling and the second $\sim 300 \mathrm{~K}$ to possible systematic errors due to the practical application of DFT, the latter inferred by comparing calculated phonon dispersions, phonon density of states as function of pressure and pressure-volume equation of states with experiments.

Recently, we have readdressed the problem using the technique of the coexistence of phases, in which solid and liquid are simulated together in a large box. This approach to melting is equivalent to the free energy approach, provided the forces on the atoms are obtained by the same potential energy method. With this method, the melting temperature of iron at $330 \mathrm{GPa}$ was calculated as $6390 \pm$ $100 \mathrm{~K}$ [14], and since the method is completely independent from the free energy approach, the close agreement of the two results strongly indicates that the DFT melting temperature of iron at $330 \mathrm{GPa}$ is in the region of $\sim 6350 \mathrm{~K}$

However accurate it may be, each implementation of DFT has one important approximation which is difficult to overcome: the exchange correlation energy (XC). This term of the potential energy contains all the physics that cannot be captured exactly. For iron, it has been shown that if the XC known as PW91 [15] is used, then a number of static and vibrational properties can be accurately predicted, including the transition pressure from the body centered cubic (bcc) phase to the hexagonal closed packed (hcp) phase $[16,17]$ - the most probable phase of iron at Earth's core conditions-, the hcp pressure versus volume equation of state, the zero pressure bcc phonon dispersions 
[18], and the high pressure hep phonon density of states [19]. However, when comparing with experimental data, small errors in the above mentioned quantities are apparent, and these errors may propagate to the calculated free energies. Although it is reasonable to expect that most of the errors due to the approximate treatment of the $\mathrm{XC}$ are similar in solid and liquid, and therefore cancel out, it is difficult to put a definite number on these uncontrollable errors.

Here we have tried to go beyond DFT, using the technique known as quantum Monte Carlo (QMC) simulation [20], and, in particular, the diffusion Monte Carlo (DMC) method, which is an exact way to solve the Schrödinger equation using a projection scheme in imaginary time which provides the ground state wave function, and therefore the ground state energy. In practice, for a fermionic system DMC cannot be applied without resorting to two approximations: the fixed node approximation (FNA), where the nodal surface of the electronic wave function is fixed to that of a predefined trial function, and the use of pseudopotentials. However, there is abundant evidence that even with the FNA, DMC is in most cases 1 order of magnitude more accurate than DFT [21,22] (albeit 3 or 4 order of magnitudes more expensive). For a description of the QMC techniques used here we refer the reader to our previous paper [23]. All present calculations were performed using the CASINO code [24]. Single particle orbitals were obtained by plane-wave DFT-PBE [25] calculations at the $\Gamma$ point in the Brillouin zone of the corresponding supercell, using the PWSCF [26] code, and reexpanded in $B$ splines [27]. We used a DFT norm-conserving pseudopotential, with atomic configuration $3 s^{2} 3 p^{6} 4 s^{1} 3 d^{7}$, and a plane-wave cutoff of 75 Hartree. Finite size errors were estimated with the method of Kwee et al. [28] as implemented by one of us (ES).

We have computed the DMC melting temperature of $\mathrm{Fe}$ at $330 \mathrm{GPa}$ by calculating free energy differences between DMC and DFT, using thermodynamic integration [29]. This is a general technique to evaluate the Helmholtz free energy difference $\Delta F$ between the two systems, given by $\Delta F=\int_{0}^{1} d \lambda\langle\Delta U\rangle_{\lambda}$, where $\Delta U=U_{\mathrm{DMC}}-U_{\mathrm{DFT}}$, with $U_{\mathrm{DMC}}$ and $U_{\mathrm{DFT}}$ the potential energies of the DMC and the DFT systems, respectively, and \langle\rangle$_{\lambda}$ represents thermal average in the ensemble generated by the potential energy function $U_{\lambda}=\lambda U_{\mathrm{DMC}}+(1-\lambda) U_{\mathrm{DFT}}$. If $U_{\mathrm{DMC}}$ and $U_{\mathrm{DFT}}$ are similar, then it is easy to show that

$$
\Delta F \simeq\langle\Delta U\rangle_{\lambda=0}-\frac{1}{2 k_{B} T}\left\langle\left(\Delta U-\langle\Delta U\rangle_{\lambda=0}\right)^{2}\right\rangle_{\lambda=0},
$$

where $k_{B}$ is the Boltzmann constant. The advantage of this perturbative approach is that we only need to compute averages in the ensemble generated by DFT. The possible drawback is that this approximation is only reliable if the second term of the right-hand side of Eq. (1), the fluctuation term, is small. To put numbers in context, we note that an error of $\sim 10 \mathrm{meV} /$ atom in the free energy difference between solid and liquid is responsible for an error of $\sim 100 \mathrm{~K}$ in the melting temperature, so we require that the size of this fluctuation term is not bigger than a few tens of $\mathrm{meV} /$ atom. The difference in melting temperature between DMC and DFT at pressure $p$ is given by [30]:

$$
\Delta T^{m} \simeq \frac{\Delta G^{l s}}{S_{\mathrm{DFT}}^{l s}}
$$

where the superscript $l s$ indicates differences between liquid and solid, $S_{\mathrm{DFT}}^{l s}$ is the DFT entropy of melting, and the difference of Gibbs free energy $\Delta G \simeq \Delta F-$ $V \Delta p^{2} / 2 K_{T}$, with $K_{T}$ the isothermal bulk modulus and $\Delta p$ the change in pressure as the potential energy is changed from $U_{\mathrm{DFT}}$ to $U_{\mathrm{DMC}}$ at constant volume $V$. The volume of interest here is about $7 \AA^{3} /$ atom, and $K_{T} \simeq$ $1300 \mathrm{GPa}$ [11], so even a difference of $20 \mathrm{GPa}$ in pressure would only be responsible for $6 \mathrm{meV}$ difference between $\Delta G$ and $\Delta F$. In fact, the difference is likely to be much smaller than that, as the DMC pressure-volume zero temperature equation of state [23] shows pressure differences between DMC and DFT of the order of 1-2 GPa. We do not have direct evidences at high temperature, or indeed for the liquid, but we believe that it would be unlikely that

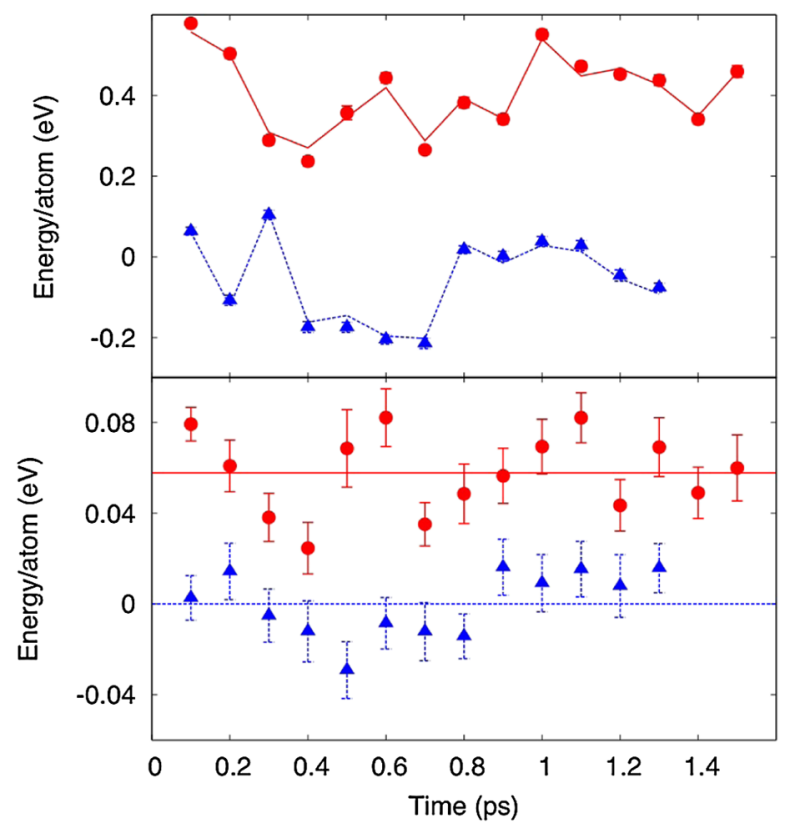

FIG. 1 (color online). Top panel: DMC energies corresponding to configurations representative of solid (blue triangles) and liquid (red dots) iron, generated with DFT molecular dynamics on 64-atom systems. Red solid line and blue dashed line connect DFT energies calculated on the same set of configurations. An offset is added to the energies so that the average value of the DMC and DFT energies is the same, separately in the solid and the liquid. Bottom panel: DMC_-DFT energy differences on the same configurations. The average DMC-DFT energy difference for the solid is subtracted from all points. Lines represent the average of the energy differences between DMC and DFT in the solid (line at zero energy) and the liquid. 
these differences would change by more than a few GPa. Moreover, most of these differences will cancel between liquid and solid, so that for present purposes the value of $\Delta F$ at constant $V$ is also representative of $\Delta G$ at constant $p$.

To compute $\Delta F$ we generated two long simulations using DFT molecular dynamics, one for the solid (in the hcp phase, with $c / a=1.6$ ) and one for the liquid, and extracted statistically independent configurations every 0.1 ps (which is twice as long as the correlation time). The simulations were performed at the DFT melting point $(p, T) \simeq(330 \mathrm{GPa}, 6350 \mathrm{~K})$ [11], with 64 atoms in the simulation cell. Using these configurations we calculated DFT and DMC energies, which are shown in the top panel of Fig. 1 for both the solid and the liquid, with an offset so that the DMC and the DFT energies have the same average value. Represented in this way, it is easy to see that the DMC energies follow quite closely the DFT energies, with the fluctuation terms being $17 \pm 7 \mathrm{meV} /$ atom and $12 \pm$ $6 \mathrm{meV} /$ atom for liquid and solid, respectively. The very low values of the fluctuation terms mean that the perturbative formula in Eq. (1) can be used reliably. In the bottom panel of Fig. 1 we show the differences between DMC and DFT energies for both solid and liquid, offset by the average of this difference in the solid. It is clear that the solid is stabilized with respect to the liquid, and we obtain the value $\Delta F^{l s}=50 \pm 20 \mathrm{meV} /$ atom, which used in Eq. (2) together with the value $S_{\mathrm{DFT}}^{l s}=1.05 k_{B}$ [11] gives a DMC correction to the DFT melting temperature $\Delta T^{m}=$ $550 \pm 230 \mathrm{~K}$. Combining this value with the DFT melting temperature $T_{\mathrm{DFT}}^{m}=6350 \pm 300 \mathrm{~K}$ (note that here we are only considering the statistical component of the DFT error, because any systematic component is absorbed as a

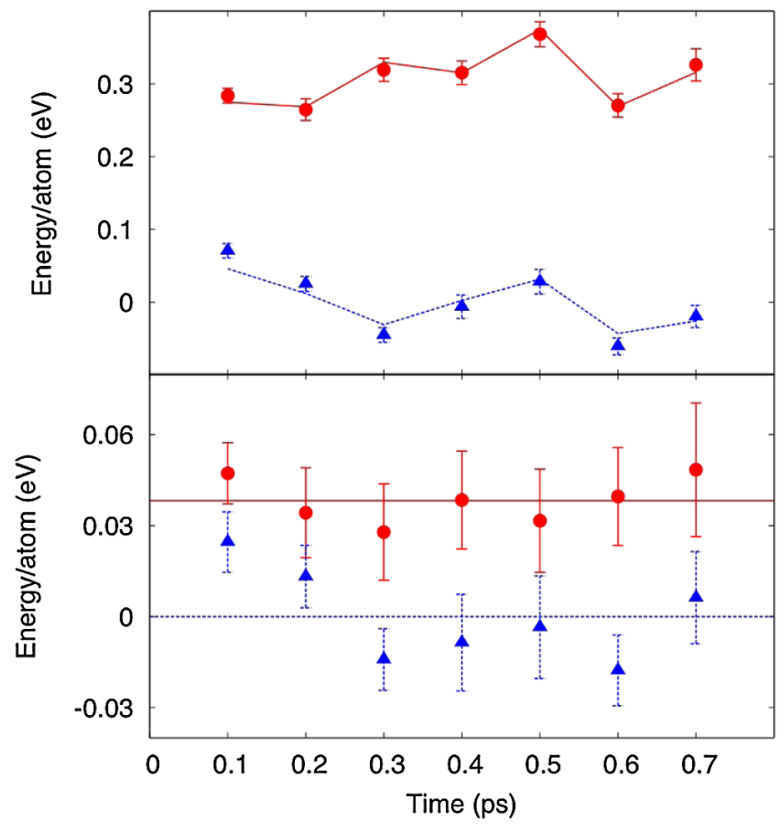

FIG. 2 (color online). Same as Fig. 1 but for 96-atom systems. constant shift in the energy differences between DMC and DFT), and considering an extra error of $\sim 100 \mathrm{~K}$ due to DMC time step errors (see below) we obtain a DMC melting temperature at $330 \mathrm{GPa}$ of $T_{\mathrm{DMC}}^{m}=6900 \pm$ $400 \mathrm{~K}$. To study possible size effects, we repeated similar calculations on systems containing 96 atoms, which we show in Fig. 2. From these simulations we obtain $\Delta F=$ $50 \pm 25 \mathrm{meV} /$ atom, which is in agreement with the results obtained with the 64-atom systems. This is expected, given the proximity of the DMC and DFT potential energy functions demonstrated by the low values of the fluctuation terms.

The present DMC melting point is reported in Fig. 3 together with other previous theoretical calculations and experimental data. Our DMC iron melting point is consistent with the shock-wave results of Brown and McQueen [7] and those of Nguyen and Holmes [8], and perhaps with the diamond anvil cell (DAC) experiments of Shen et al. [4] and Ma et al. [6], though these experiments are only available to much lower pressures. This melting temperature is difficult to reconcile with any reasonable extrapolation of the DAC experiments of Boehler [3]. The differences between the theoretical melting curves reported in Fig. 3 have been discussed in Ref. [14], and are due to the fact that the calculations of Refs. $[12,13]$ were based on classical potentials fitted to DFT calculations.

How reliable are the present DMC results? There are two kind of errors in the calculations. Those of the first kind are related to convergence with respect to various technical parameters. Specifically, these are DMC time

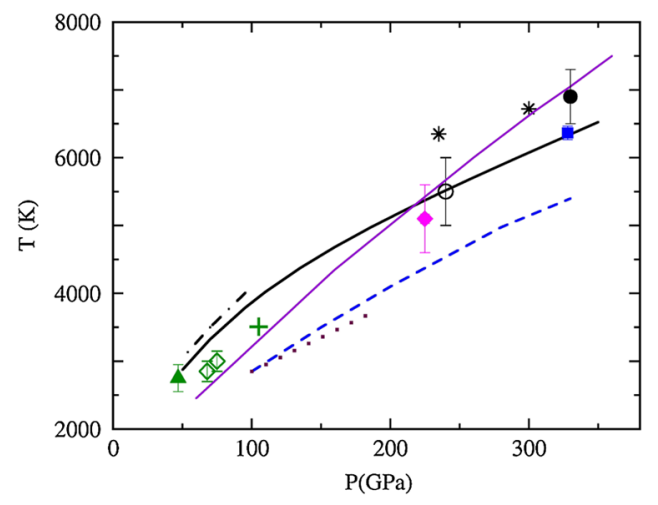

FIG. 3 (color online). Comparison of melting temperatures (T) of $\mathrm{Fe}$ as function of pressure $(\mathrm{P})$ from present calculations with experiments and other $a b$ initio results. Black filled circle: present DMC results; blue filled square: melting point from DFT coexistence [14]; solid black line: melting curve from DFT free energies [11]; dashed blue curve: theoretical results from Ref. [13]; light purple solid curve: theoretical results from Ref. [12]; black chained and maroon dotted curves: DAC measurements of Refs. [2,3]; green open diamonds: DAC measurements of Ref. [4]; green plus: DAC measurement of Ref. [6]; green filled triangle: DAC measurement of Ref. [5]; black stars, black open circle and pink filled diamond: shock experiments of Refs. [7-9]. Error bars are those quoted in original references. 
step errors, DMC finite size errors (in addition to the finite size errors discussed above), and DMC statistical errors. DMC time step errors have been investigated by repeating simulations with time steps of $0.02,0.01,0.005$, 0.0025 and 0.00125 a.u. on two representative configurations, one for the liquid and one for the solid. These tests showed that using a time step of 0.01 a.u. the relative error between solid and liquid is less than $10 \mathrm{meV} /$ atom. DMC finite size errors are of the order of 0.23 and $0.15 \mathrm{eV} /$ atom for the 64-atom and the 96-atom systems, respectively, but they are essentially identical in solid and liquid-with relative differences of less than $3 \mathrm{meV} /$ atom-, so these errors are completely negligible. Finally, DMC simulations were run for 2500-5000 steps, resulting in errors between 10 and $15 \mathrm{meV}$ for each DMC energy point. All these errors have been statistically combined in the error estimates reported above, a part from the (small) finite size errors which have been treated as rigid free energy shifts. The second kind of errors are related to the FNA and the use of pseudopotentials. The close agreement with the experiments for the DMC pressure-volume equation of state [23] indicates that at least for the zero temperature solid the combination of these two errors is indeed extremely small, and confirms the general trend of high accuracy of DMC even with single Slater determinant trial functions.

In conclusion, we have presented $a b$ initio parameter free diffusion Monte Carlo free energy calculations for iron at Earth's core conditions, which we have used to obtain a melting temperature at the inner core boundary pressure of $T_{\mathrm{DMC}}^{m}=6900 \pm 400 \mathrm{~K}$. This melting temperature is slightly higher than that obtained with DFT, and combined with the estimated depression of melting temperature due to the presence of light impurities [31] gives a probable core temperature of $6000 \pm 500 \mathrm{~K}$. This raised core temperature also raises the adiabatic gradient throughout the core, and therefore the heat flux lost by conduction, possibly leading to a shorter life of the inner core (less than the preferred age of $3.5 \times 10^{9}$ years [32]). It will also be responsible for a higher coremantle-boundary (CMB) temperature of over $4400 \mathrm{~K}$, which might be higher than the solidus at the CMB and therefore result in partial melting of the bottom of the mantle, and could explain the presence of ultra-lowvelocity zones.

This work was supported by a EURYI scheme as provided by EPSRC (see www.esf.org/euryi) and by NERC. Calculations were performed on the UK national facility HECToR, typically on 1024 cores, each DMC point taking $\sim 100$ hours and $\sim 150$ hours for the 64-atom and the 96atom systems, respectively, using allocation of time from NERC through the Mineral Consortium and from a EPSRC Capability Challenge grant. Calculations were also performed on the UCL research-computing facility Legion, and initially on the Cambridge High Performance facility Darwin. We thank D. Gubbins, D. Dobson, G. D. Price, and M. J. Gillan for useful discussions.
[1] J.-P. Poirier, in Introduction to the Physics of the Earth's Interior (Cambridge University Press, Cambridge, England, 1991).

[2] Q. Williams, R. Jeanloz, J. D. Bass, B. Svendsen, and T. J. Ahrens, Science 236, 181 (1987).

[3] R. Boehler, Nature (London) 363, 534 (1993).

[4] G. Shen, H. Mao, R. J. Hemley, T. S. Duffy, and M.L. Rivers, Geophys. Res. Lett. 25, 373 (1998).

[5] A. P. Jephcoat and S. P. Besedin, Phil. Trans. R. Soc. A 354, 1333 (1996).

[6] Y. Ma et al., Phys. Earth Planet. Inter. 143-144, 455 (2004).

[7] J. M. Brown and R. G. McQueen, J. Geophys. Res. 91, 7485 (1986)

[8] J. H. Nguyen and N. C. Holmes, Nature (London) 427, 339 (2004).

[9] C. S. Yoo, N. C. Holmes, M. Ross, D. J. Webb, and C. Pike, Phys. Rev. Lett. 70, 3931 (1993).

[10] D. Alfè, M. J. Gillan, and G. D. Price, Nature (London) 401, 462 (1999).

[11] D. Alfè, G. D. Price, and M. J. Gillan, Phys. Rev. B 65, 165118 (2002).

[12] A. B. Belonoshko, R. Ahuja, and B. Johansson, Phys. Rev. Lett. 84, 3638 (2000).

[13] A. Laio, S. Bernard, G. L. Chiarotti, S. Scandolo, and E. Tosatti, Science 287, 1027 (2000).

[14] D. Alfè, Phys. Rev. B 79, 060101(R) (2009).

[15] Y. Wang and J. P. Perdew, Phys. Rev. B 44, 13298 (1991).

[16] L. Stixrude, R. E. Cohen, and D. J. Singh, Phys. Rev. B 50, 6442 (1994).

[17] P. Söderlind, J. A. Moriarty, and J. M. Wills, Phys. Rev. B 53, 14063 (1996).

[18] D. Alfè, G. Kresse, and M. J. Gillan, Phys. Rev. B 61, 132 (2000).

[19] H. K. Mao et al., Science 292, 914 (2001).

[20] B.L. Hammond, W. A. Lester, Jr., and P. J. Reynolds, Monte Carlo Methods in Ab Initio Quantum Chemistry (World Scientific, Singapore, 1994) p. 1004.

[21] G. Rajagopal, R. J. Needs, A. James, S.D. Kenny, and W. M. C. Foulkes, Phys. Rev. B 51, 10591 (1995).

[22] M. Pozzo and D. Alfè, Phys. Rev. B 77, 104103 (2008).

[23] E. Sola, J.P. Brodholt, and D. Alfè, Phys. Rev. B 79, 024107 (2009).

[24] R. J. Needs, M. D. Towler, N. D. Drummond, and P. Lopez Rios, CASINO version 2.1 User Manual (University of Cambridge, Cambridge, England, 2007).

[25] J. P. Perdew, K. Burke, and M. Ernzerhof, Phys. Rev. Lett. 77, 3865 (1996).

[26] S. Baroni, A. Dal Corso, S. de Gironcoli, and P. Giannozzi, http://www.pwscf.org.

[27] D. Alfè and M. J. Gillan, Phys. Rev. B 70, 161101 (2004).

[28] H. Kwee, S. Zhang, and H. Krakauer, Phys. Rev. Lett. 100, 126404 (2008).

[29] D. Frenkel and B. Smit, Understanding Molecular Simulation: From Algorithms to Applications (Academic Press, San Diego, 1996).

[30] D. Alfè, M. J. Gillan, and G. D. Price, J. Chem. Phys. 116, 7127 (2002).

[31] M. J. Gillan, D. Alfè, J. Brodholt, L. Vočadlo, and G. D. Price, Rep. Prog. Phys. 69, 2365 (2006).

[32] D. Gubbins, D. Alfè, G. Masters, G. D. Price, and M. J. Gillan, Geophys. J. Int. 157, 1407 (2004). 